\title{
Führung, Kooperation und Networking
}

\author{
AK Organisation der Schmalenbach-Gesellschaft für \\ Betriebswirtschaft e.V.*
}

Die Corona-Pandemie hat zum verstärkten Einsatz von digitalen Technologien in Unternehmen geführt, die das Zusammenwirken, die Führung und Kooperation deutlich verändert haben. Anstatt die organisatorische Intelligenz in immer anspruchsvolleren formalen Strukturen und Prozessen zu verwirklichen, wie es traditionell dem organisatorischen Gestaltungsansatz entspricht, verzeichnen alle Unternehmen im AK Organisation der Schmalenbach-Gesellschaft für Betriebswirtschaft e.V. einen Trend zu mehr teamorientierten Organisationen, die ihre Arbeit mit Hilfe von Digitaltechnologien koordinieren.

1. These: Die traditionelle Vorstellung einer starken Führung durch eine designierte Führungselite, die ihr Einflusspotential insbesondere aufgrund ihrer Position in der Autoritätshierarchie geltend macht, wird zugunsten einer Perspektive von Führung als Rahmensetzer, Facilitator, Coach und Ermöglicher ersetzt.

Durch die Corona-Pandemie hat die Digitalisierung der Arbeitsprozesse bei den Arbeitskreisunternehmen einen deutlichen Sprung nach vorne gemacht. Damit werden heutzutage sehr viele Arbeitsplätze durch den umfangreichen Einsatz von digitalen Technologien geprägt und erfordern von Führungskräften und Mitarbeitenden neue Fähigkeiten und neue Formen der Zusammenarbeit. In erster Linie ist Führung im digitalen Kontext dafür verantwortlich, vernetzte, offene und transparente Arbeitsumgebungen über alle hierarchischen Ebenen und Organisationseinheiten hinweg zu schaffen. Damit werden die informationellen und organisatorischen Voraussetzungen dafür geschaffen, dass die Organisationsmitglieder ihre Aufgaben stärker im Team sowie unter Rückgriff auf digitale Technologien

AK Organisation | Schmalenbach-Gesellschaft für Betriebswirtschaft e.V. | sg@schmalenbach.org

Federführende Autoren: Michael Kowarsch, Covestro AG | Dr. Neele Wieczorek, Volkswagen AG | Dr. Michael Schäffer, Siemens AG | Dr. Friedrich-Wilhelm Janssen, EWE AG | Prof. Dr. Markus Reihlen, Leuphana Universität Lüneburg | Prof. Dr. Michèle Morner, Universität Speyer. 
und verteilte Informationen selbst organisieren können. So hat EWE beispielsweise mit Ausbruch der Pandemie die Arbeitsgruppe „New Normal“ geschaffen, die sich mit Leitplanken während der Coronakrise auseinandergesetzt hat. Diese Leitplanken wurden dann in Rahmen von digitalen Workshops in allen Organisationseinheiten diskutiert, um letztlich die digitale Vernetzung in den Teams und zu anderen Abteilungen zu stärken.

Führungskräften kommt in diesem Kontext eine veränderte Rolle zu, denn die traditionelle Vorstellung einer starken Führung von oben, die ihr Einflusspotential insbesondere aufgrund ihrer hierarchischen Position begründet, wirkt nicht mehr zeitgemäß. Führung in digitalen Arbeitswelten wird stattdessen anders gedacht: Ihre Aufgabe besteht darin, eine Anleitung zur Selbststeuerung zu geben. Insgesamt verändert sich damit das Rollenverständnis der Führungskräfte, die sich vor diesem Hintergrund eher als Rahmensetzer, Facilitator, Coach und Ermöglicher begreifen und nur im Ausnahmefall zum Mittel der direkten Intervention greifen sollten. Entsprechend hatte Covestro bereits vor Ausbruch der Pandemie damit begonnen, seine Identität und Kultur weiterzuentwickeln, indem ein Zielbild „What Makes Us Covestro" (WMUC) mit zwölf wünschenswerten Verhaltensweisen beschrieben wurde. Das Zielbild für die Führung deckt sich mit der Idee des Coaches, Rahmensetzers und Facilitators. Diese „neue Art" der Führung, unterstützt durch digitale Tools, haben bei allen Arbeitskreisunternehmen an Bedeutung gewonnen.

2. These: Führung im digitalen Arbeitskontext wird einerseits egalitärer und andererseits situativer.

Die Erfahrungen der Mitarbeitenden unterschiedlicher Hierarchieebenen zeigen, dass virtuelle Austauschformate als gleichberechtigtere Partizipationschancen wahrgenommen werden. Führungspersonen werden über digitale Kommunikationsformate nahbarer. Zudem lassen sich Führungsprozesse leichter und schneller digital organisieren, indem (weltweit) alle Personen, die für Entscheidungsprozesse substantielles Wissen beitragen können, eingebunden werden. Dadurch wird das Phänomen der „Hinterzimmergespräche“ und „Nebenabsprachen" gemindert. Covestro hat beispielsweise zu Beginn der Pandemie verschiedene digitale Austauschformate eingeführt. Dazu zählen wöchentliche „Weare1-Talks“, in denen zu Beginn der Vorstandsvorsitzende und mittlerweile alle Vorstände in einem Video über aktuelle Entwicklungen informieren. Zusätzlich können anonym Fragen über ein digitales Tool gestellt werden. Sechs Mal im Jahr werden "globale townhall“-Formate angeboten, indem alle Mitarbeitenden weltweit zu einem gemeinsamen Meeting mit dem Vorstand zusammenkommen. Im Ergebnis empfinden sich Mitarbeitende in digitalen Austauschformaten mehr als gleichwertige Gesprächspartner. Andererseits berichten Führungskräfte, dass die Führung zugleich situativer erfolgen muss. So versucht man bei VW auf individuelle Bedürfnisse der Mitarbeitenden einzugehen, die durch die Pandemie unterschiedlich belastet sind. Dies erfolgt beispielsweise durch Kinderbetreuung, digitale aber auch physiche Arbeitsplätze oder durch flexible Arbeits- und Urlaubszeitmodelle. Da sich sowohl die Situation der Mitarbeitenden - z.B. Erfahrungs- und Integrationsgrad im Team oder Arbeitsbedingungen im Homeoffice - als auch die Aufgabenkontexte schnell ändern können, bedarf es situativer Führungsinterventionen. 
3. These: In rein virtuellen Arbeitskontexten wird die Integration der Mitarbeitenden und die Identifikation mit dem Unternehmen deutlich erschwert, wodurch Motivations- und Abstimmungsprobleme im Arbeitsalltag entstehen können.

Die Integration der Organisationsmitglieder erfolgt über Sozialisierungs-, Ausbildungs- und informelle Austauschprozesse, die zu einer stärkeren Identifikation der Mitarbeitenden mit dem Unternehmen beitragen sollen. Eine solche Identifikation vermittelt ein Zusammengehörigkeitsgefühl und gibt Orientierung für zentrale Identitätsfragen, wie beispielsweise: „Wer sind wir als Unternehmen?" und „Wo wollen wir zukünftig stehen?". Ein geteiltes Verständnis der Organisation bzgl. ihrer Ziele und Ambitionen ist eine wesentliche Voraussetzung, um Selbstorganisation in produktive Bahnen zu lenken. Durch die virtuellen Arbeitsbeziehungen wird dies jedoch erschwert, weil hier kaum starke Integrations- und Identifikationsmechanismen wirken können. Dies führt nach den Erfahrungen der Arbeitskreisunternehmen immer dann zu Problemen in der Zusammenarbeit, wenn zukünftige Entwicklungsperspektiven nicht geteilt werden, mangelndes Vertrauen die Koordination erschwert oder das Arbeiten in vielen unterschiedlichen virtuellen Arbeitszusammenhängen austauschbar wird. Bei VW wurde insbesondere das Onboarding neuer Mitarbeitender als Herausforderung gesehen. Aber auch das Führen kritischer Personalgespräche sowie das strategische und kreative Arbeiten im Team wird nur bedingt durch digitale Tools sinnvoll unterstützt. Als Reaktion auf diese Integrations- und Identifikationsprobleme haben alle Arbeitskreisunternehmen mit neuen digitalen Formaten experimentiert. So wird das Onboarding neuer Kolleginnen und Kollegen durch zahlreiche neue digitale „social Meeting"-Formate wie Kick-off's, Coffee Bars, virtuelle Lunch oder Dinner Meetings unterstützt. Zudem wurden häufigere und kürzere Check-in Meetings bei der Tätigkeit im Homeoffice eingeführt. Mit diesen neuen Sozialisierungsformaten, die früher stillschweigend im Arbeitsalltag integriert waren und quasi nebenher liefen, steigt zugleich nun auch der Zeitaufwand für die Führung. So müssen in virtualisierten Arbeitszusammenhängen informelle Zusammenkünfte extra organisiert werden. Zusätzlich versucht man bei Covestro diesen Herausforderungen durch identitätsbezogene Maßnahmen zu begegnen. So wurde bereits vor Ausbruch der Pandemie der Slogan „Weare1“ kreiert, der das Zusammengehörigkeitsgefühl und die Identifikation mit dem Unternehmen über unterschiedliche Gruppen hinweg stärken sollen.

4. These: Virtuelle Kooperation führt zu einer Vernetzungsparadoxie: Einerseits dehnt sich die Reichweite der Vernetzungsmöglichkeiten aus, da die Kosten der virtuellen im Vergleich zur physischen Vernetzung minimal sind. Andererseits nehmen die Tiefe, Vertrautheit und das wechselseitige Verständnis mit zunehmender Reichweite ab.

Die Arbeitskreisunternehmen teilen ähnlich positive Erfahrungen bei der virtuellen Zusammenarbeit bestehender Teams, die bereits auf eine erfolgreiche Historie und Kultur der Kooperation aufbauen können. Hier wurde bereits Sozialkapital geschaffen, welches innerhalb der Gruppen integrierend wirkt und somit zu Loyalität und wechselseitiger Unterstützung der Teammitglieder untereinander beiträgt. Solche bereits bestehenden Teams, und dies zeigen die Erfahrungen aus dem Arbeitskreis, 
haben durch den Wechsel in einen rein virtuellen Arbeitsmodus kaum Motivations- und Produktivitätseinbußen zu verzeichnen. Die Vernetzung mit neuen Personen, Abteilungen und Organisationen ist hingegen bei einer rein virtuellen Kooperation wesentlich herausfordernder, da das integrierende Sozialkapitel noch fehlt. Zudem zeigen die Erfahrungen, dass die Schaffung vertrauensvoller neuer Arbeitszusammenhänge durch rein digitale Austauschformate erschwert wird. Ein differenzierter Blick aus Sicht der Erfahrungen der Arbeitskreisunternehmen weist jedoch darauf hin, dass sich zwei Fälle unterscheiden lassen. Einerseits wird die Schaffung von transaktionalen Beziehungen mit neuen Partnern durch die Digitalisierung erleichtert, da sehr schnell und flexibel globale Partner integriert werden können. Dafür müssen aber Schnittstellen und Leistungen für den Leistungsaustausch klar definiert sein. Die größere Reichweite der digitalen Vernetzung kann dann dazu beitragen, innovativere oder preisgünstigere Komponenten in die Leistungsbereiche zu integrieren. Andererseits wird die Netzwerkbildung erschwert - sofern diese über eine rein transaktionale Beziehung hinaus geht. Dies ist beispielweise dann der Fall, wenn komplexe Innovationsprozesse mit neuen Partnern stattfinden, die letztlich ein tieferes, wechselseitiges „Integrationswissen" erfordern. Dieses Integrationswissen wird nur unzureichend über virtuelle Austauschformate vermittelt. Diesem Problem versucht man bei EWE durch die Initiative „New Normal (nach Corona)“ zu begegnen. Ziel ist es, eine gute Balance zwischen neuer und alter Welt zu finden. Im Ergebnis führt die Virtualisierung der Arbeitszusammenhänge dazu, dass bestehende Netzwerke gestärkt werden und neue Verbindungen hingegen zunächst rein transaktionalen Charakter haben, da die Entwicklung tieferer, vertrauensvoller Beziehungen nur unzureichend durch digitale Medien hergestellt werden kann.

Quelle

Der Debattenbeitrag basiert auf der Diskussionsreihe „Lehren aus der virtuellen Zusammenarbeit: Das new normal nach COVID." im AK Organisation der Schmalenbach-Gesellschaft für Betriebswirtschaft e.V. 